Available online at_www.iponlinejournal.com

\title{
Silver nanoparticles used in the antibacterial preparations
}

\author{
Chandni Yadav ${ }^{1 *}$, Vaishnav Rajat ${ }^{2}$, Anju Goyal $^{3}$ \\ ${ }^{1,2}$ Assistant Professor, ${ }^{3}$ Professor and HOD, Dept. of Quality Assurance, Bhupal Noble's Institute of Pharmaceutical Sciences, \\ B.N. University, Udaipur, Rajasthan, India
}

\begin{abstract}
Nanotechnology is the emerging field in medicine that is expected to induce exceptional therapeutic benefits. Nanoparticles have been improving the therapeutic effect of drugs and are prepared by using varioustechniques. Silver Nanoparticles are attracting interest for the clinical application because of its potential biological properties such as antibacterial properties; antifungal properties etc. Due to its highly efficient properties Silver Nanoparticles are invasively used in the field of medical and health care. Against a large number of micro-organisms, Silver Nanoparticles are proven to be exceptionally productive for their antibacterial effects. Its mechanism of action is still unknown but the method of synthesis is highly economical, affordable and reliable. Desired properties can be achieved by changing the shapes and Sizes of Silver Nanoparticles.

The main aim of this work is to provide an outline and general review on Silver Nanoparticles, with special reference to their mechanism of action and antimicrobial activity.
\end{abstract}

Keywords: Silver nanoparticles, Antimicrobial activity, Biocompatibility, Synthesis, Medical applications.

\section{Introduction}

Nanotechnology is the emerging field of science that incorporates synthesis and development.

Basically Nanoparticles is defined as the objects that fluctuate in size ranging from 1-100 nm and can also vary in size that of the bulk material.

Recently various metallic nano materials are evolved using copper, zinc, titanium, magnesium, gold, alginate and Silver. Nanoparticles are diversified for numerous purposes, from medical treatments to utilize in various branches of industry production. ${ }^{1}$

\section{Synthesis of nanoparticles}

Nanoparticles may be synthesized chemically or biologically. Various adverse effects have been affiliated with chemical synthesis methods due to the existence of some toxic chemical sop up on the surface. Eco-friendly alternatives to chemical and physical methods are biological ways of Nanoparticles synthesis using micro-organisms, enzymes, fungus and plants or plant extracts.
The development of these co-friendly methods for the synthesis of Nanoparticles is evolving into an important branch of nanotechnology especially silver Nanoparticles, which have many applications. ${ }^{2,3}$

\section{Silver nanoparticles}

Over the last decades, Silver Nanoparticles, objects from 1 to $100 \mathrm{~nm}$ in size, were extensively investigated due to their unique size-dependent optical, electrical and magnetic properties. Silver Nanoparticles are mostly used for their antimicrobial and antifungal properties. Numerous synthesis were being developed to carry out the production of the Silver Nanoparticles but the major objective was to carry out the synthesis of the silver Nanoparticles with an efficient anti-microbial properties in an easy and cost effective way. Now-a-days, Silver Nanoparticles are invasively used in varied fields, inclusive medical, food, health care, consumer, and industrial purposes, due to their unique physical and chemical properties. These include optical, electrical, and thermal, high electrical conductivity, and biological properties. ${ }^{4-6}$

\footnotetext{
*Corresponding Author: Chandni Yadav, Dept. of Quality Assurance, Bhupal Noble's Institute of Pharmaceutical Sciences, B.N. University, Udaipur, Rajasthan, India

Email: chandniyadav093@gmail.com

http://doi.org/10.18231/j.ijpca.2019.011
} 
Due to their distinctive properties, they have been used for various applications, including as antibacterial agents. Against a wide range of microorganism, Silver Nanoparticles is proven to have excellent bactericidal The structures of Silver Nanoparticles exhibit significantly novel and distinct physical, chemical, and biological properties and functionality due to their Nanoparticles size, have extracted much interest. Their potential for achieving specific processes and selectivity in the biological sectors are the major point of attention. ${ }^{9}$

There are various properties of Silver Nanoparticles and are as follows:

1. Throughout the history of civilization, Silver Compounds are been used in the medicine. ${ }^{10}$

2. Silver Nanoparticles can be easily incorporated into various cotton fabrics and dressings. ${ }^{11}$

3. Silver Nanoparticles have an efficient antiinflammatory property which promotes wound healing. ${ }^{12}$

4. Silver Nanoparticles are easily synthesized by affordable and reliable methods such as wet chemical, physical, and biological. ${ }^{13}$

5. Silver Nanoparticles shows an efficient antibacterial effect against a large no. of microorganisms. ${ }^{14}$

6. Silver Nanoparticles can be synthesized on various shapes and sizes. ${ }^{13}$

7. Bacterial resistance to elemental silver is extremely rare, suggesting the presence of multiple bactericidal mechanisms acting in synergy. ${ }^{15}$

8. Silver Nanoparticles are highly reactive. ${ }^{13}$

\section{Benefits of silver nanoparticles}

Silver Nanoparticles has many benefits, some of them are mentioned as below:

1. The organism's chances of developing resistance are considerably reduced by the multilevel antibacterial effects on the cell.

2. Silver Nanoparticles have high potency against multi-drug-resistant organisms.

3. Silver Nanoparticles have a low systemic toxicity

Although, other Silver compounds such as silver nitrate and silver sulfadiazine are variably used for properties. ${ }^{7}$ They are used in catheters, cuts, burns and wounds to protect them against infection ${ }^{8}$

\section{Properties of silver nanoparticles}

topical applications as they may be neutralized by anions in body fluids or cause cosmetic abnormality on prolonged use, and they can hinder the healing process via fibroblast and epithelial cell toxicity. Despite all the other silver compounds, silver sulfadiazine is the most desired topical antimicrobial silver delivery system in use because safer alternatives are not available. ${ }^{16}$

\section{Mechanism of action}

The recent exposure of nanotechnology has provided a new therapeutic mode in silver Nanoparticles for use in various wounds. The effect of Silver Nanoparticles in dermal contraction and epidermal reepithelization during wound healing and suggested that silver Nanoparticles could enhance the rate of wound closure. Basically this was achieved through the promotion of proliferation and migration of keratinocytes. ${ }^{17}$ On the other hand, silver Nanoparticles could drive the differentiation of fibroblasts into Myofibroblasts, thereby promoting wound contraction. Silver Nanoparticles play a distinct role in preventing infection and decreasing bacterial impact in the wound by their broad-spectrum antimicrobial properties, and their surfacemodification properties providing simple incorporation of Nano silver into cotton fabrics and drugs to upgrade the wound-healing treatment. Along with the above properties, the potent anti-inflammatory properties of Nano silver mediated through cytokine modulation lead to better therapeutic direction in wound treatment.

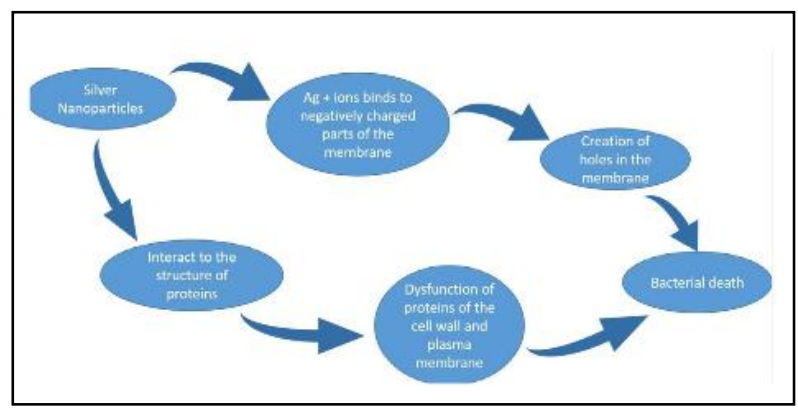

Fig. 1: Antibacterial mechanism of silver nanoparticles ${ }^{17}$ 


\section{Antibacterial activity of silver nanoparticles}

Because of the development of antibiotic resistance to different pathogenic bacteria of various infectious diseases, many pharmaceutical companies and researchers are searching for new antibacterial agents that do not invite resistance and even are low in cost. In today's scenario, an agent must possess good physical and chemical properties, high ratio of surface area to volume, good electronic and Stearic properties. Looking forward to these factors Silver Nanoparticles have emerged as novel antimicrobial agents, owing to their high ratio of surface area to volume and their unique chemical and physical properties. Having low toxicity to human cells, high thermal stability and low volatility, Silver Nanoparticles can be used in various fields, particularly medicine and pharmaceuticals. ${ }^{15}$ Silver Nanoparticles have played a role as drugs and as superior antimicrobial agents and have even been shown to prevent HIV binding to host cells. ${ }^{18}$

Silver Nanoparticles exhibit antibacterial effects against a large number of bacterial species. The mechanisms of action and binding of silver Nanoparticles to microbes is yet unclear, but still the three principal mechanisms for its activity are known to believe as:

1. Silver binds to the bacterial cell wall and cell membrane and inhibits the respiration process ${ }^{20}$ by which the chemical energy of molecules is released and partially captured in the form of adenosine triphosphate (ATP)

2. Silver Nanoparticles interact with sulfurcontaining proteins of the bacterial membrane, as well as with phosphorus-containing compounds such as DNA, to inhibit replication. ${ }^{19}$

3. The bactericidal effect of silver has also been attributed to inactivation of the enzyme phosphomannose isomerase, ${ }^{[20]}$ which catalyzes the conversion of mannose-6-phosphate to fructose-6-phosphate, an important intermediate of glycolysis, the most common pathway in bacteria for sugar catabolism.

\section{Methods to improve biocompatibility of silver nanoparticles}

Silver Nanoparticles have various applications and therefore the biocompatibility should be paid heed for medical uses. Basically there are three methods to enhance the biocompatibility of Silver Nanoparticles.

\section{Biosynthesis to improve biocompatibility}

Mostly chemical and physical methods are widely used for Silver Nanoparticles synthesis. However, these methods have resulted in associated risks, such as chemical precursor contamination, solvent toxicity, and hazardous byproduct formation, which make alternative synthetic methods essential. ${ }^{21}$ Currently, bio-enthused synthesis of Nanoparticles are more advantageousthan chemical and physical methods as it is environment-friendly, no need to use high-pressure, high temperature, and no toxic chemicals are needed in biological method. ${ }^{22}$

Various resources can be used in the synthesis of Nanoparticles such as bacteria, yeast, fungi, and various parts of plants. Plant extracts are more advantageous for biosynthesis and offer good manipulation and control over crystal growth and stabilization. ${ }^{23}$ In most cases, there are various metabolites that can be used to reduce $\mathrm{Ag}^{+} \cdot{ }^{24} \mathrm{By}$ adjustment reaction parameters, Silver Nanoparticles can achieve better yield, controlled size, shape, greater particle stability, more biocompatibility, scalability, and applicability. ${ }^{21}$

In conclusion, biosynthesis is a promising method to produce Silver Nanoparticles with both remarkable antibacterial effect and biocompatibility.

\section{Adjustment of physical properties}

The elemental feature of metallic Nanoparticles strongly rely on their shapes, sizes, configurations, crystallinity, and structure whether they are in solid form or in hollow geometries. Therefore, by adjusting such parameters Nanoparticles can achieve the desired properties. $^{25}$

Studies have highlighted the role of size and shape in potentiating Silver Nanoparticles based cellular effect. Studies also illustrated that smaller Silver Nanoparticles shows better antimicrobial activity. The shape of Silver Nanoparticles can influence the degree of particle toxicity. In order to systematically explore these facts, spherical, rod-shaped, truncated triangular Silver Nanoparticles, and their skin penetration capabilities were deliberated. From the conclusion, it 
was noted that triangular Silver Nanoparticles could be an ideal candidate for topical applications which can reduce systemic toxicity, compared to the rod-shaped and spherical Silver Nanoparticles. ${ }^{26}$ Regardless of the size required, narrow size distributions and more "spherical" shapes can improve sample quality. ${ }^{27}$

\section{Combination with biomolecules}

Surface modification of nano materials is imperative where the surface layer facilitates the reduction of surface energy. It can also provide a protective coating that prevents Nanoparticles from agglomeration, thus increasing their long-term stability. ${ }^{28}$ Many organic molecules can be used for surface functionalization. Examples include small molecules like lipids, vitamins, peptides, and sugar and larger ones such as natural polymers including proteins, enzymes, DNA, and RNA. ${ }^{28}$

\section{Other medical applications of silver nanoparticles}

Silver Nanoparticles have applications in the diagnosis and treatment of cancer, and are drug carriers that can deliver therapeutic agents, which are used in eye care for coating contact lenses. In addition, the use of nano silver in combination with vanadium oxide in battery cell components is one instance of advanced silver nanotechnology improving battery performance in next-generation active implantable medical devices. ${ }^{29}$

\section{Conclusion and future perspectives}

An effective and complete process of wound healing is critical for the general well-being of any patients. In recent times, tremendous progress has been made in discovering the cellular and molecular mechanisms underlying the wound healing process. In current clinical treatments of wounds and ulcers, medications such as topical antimicrobial agents are still relevant. Moreover, applying nanotechnology and incorporating knowledge of cellular, subcellular events occurring during the typical healing process, could obviously get better future therapeutic interventions. Nanotechnology offers great opportunities for improving wound treatments. The nanometer scale opens the way for the development of novel materials for use in highly advanced medical technology. Silver Nanoparticles exhibit remarkable biological properties, such as anti-inflammatory, antiviral activities and antibacterial properties with less bacterial resistance. Silver Nanoparticles dressings are now the new gold standard in the conservative treatment of wounds and burns. The full potential of this technology has yet to be discovered. The mechanisms underlying the impressive wound-healing properties of silver Nanoparticles are still not understood, and understanding them is a priority for future research in vivo.

\section{Source of Funding}

None.

\section{Conflict of Interest}

None.

\section{References}

1. Dubchak S, Ogar A, Mietelski J.W. and Turnau K, Influence of silver and tianium nanoparticles on arbuscularmycorhiza colonization and acumulation of radiocaesium in Helianthus anus, Span J Agric Res 2010;8(1):103-8.

2. Klaus T, Joerger R, Olsson E. and Granqvist C.G, SilverBased Crystalline Nanoparticles, Microbially Fabricated, $J$ Proc Natl Acad Sci USA, 1999;96:13611-4.

3. Konishi Y. and Uruga T, Bioreductive Deposition of Platinum Nanoparticles on the Bacterium Shewanella algae, J Biotechnol 2007;128:648-53.

4. Gurunathan S, Park J.H, Han J.W, Kim J.H. Comparative assessment of the apoptotic potential of silver nanoparticles synthesized by Bacillus tequilensis and Calocybeindica in MDA-MB-231 human breast cancer cells: Targeting p53 for anticancer therapy. Int J Nanomed 2015;10:4203-4222. doi:10.2147/IJN.S83953.

5. Li W.R, Xie X.B, Shi Q.S, Zeng H.Y, Ou-Yang Y.S, Chen Y.B. Antibacterial activity and mechanism of silver nanoparticles on Escherichia coli. Appl Microbiol Biotechnol 2010;8:1115-22. doi: 10.1007/s00253-0092159-5.

6. Mukherjee P, Ahmad A, Mandal D, Senapati S, Sainkar S.R, Khan M.I et al. Fungus-mediated synthesis of silver nanoparticles and their immobilization in the mycelial matrix: A novel biological approach to nanoparticle synthesis. Nano Lett 2001;1:515-519. doi: 10.1021/n10155274.

7. Siddiqi KS, Husen A, and Rifaqat A. K. Raoet. Al. A review on biosynthesis of silver nanoparticles and their biocidal properties 2018;16:14. doi: 10.1186/s12951-0180334-5 
8. Crabtree JH, Burchette RJ, Siddiqi RA, Huen IT, Handott LL, Fishman A et al. The efficacy of silver-ion implanted catheters in reducing peritoneal dialysis-

9. Patra C.R, Bhattacharya R, Wang E. Targeted delivery of gemcitabine to pancreatic adenocarcinoma using cetuximab as a targeting agent. Cancer Res 2008;68:1970-8.

10. Klasen H.J. Historical review of the use of silver in the treatment of burns: early uses. Burns 2000;26:117-30.

11. Castillo P.M., Herrera J.L., Fernandez-Montesinos R. Tiopronin monolayer-protected silver nanoparticles modulate IL-6 secretion mediated by Toll-like receptor ligands. Nanomedicine 2008;3:627-35.

12. Boucher W., Stern J.M., Kotsinyan V. Intravesicalnanocrystalline silver decreases experimental bladder inflammation. J Urol 2008;179:1598-1602.

13. Patra C.R, Bhattacharya R, Wang E. Targeted delivery of gemcitabine to pancreatic adenocarcinoma using cetuximab as a targeting agent. Cancer Res 2008;68:1970-8.

14. Silver S. Bacterial silver resistance: molecular biology and uses and misuses of silver compounds. FEMS Microbiol Rev 2003;27:341-53.

15. Shrivastava $S$. Characterization of enhanced antibacterial effects of novel silver nanoparticles. Nanotechnol 2007;18:225103-225112.

16. Gunasekaran T, Nigusse T, and Dhanaraju MD, Silver Nanoparticles as Real Topical Bullets for Wound Healing. J Am Coll Clin Wound Spec 2011;3(4):82-96. doi: 10.1016/j.jcws.2012.05.001

17. Lee P.Y, Ho C.M, Lui V.C.H. Silver nanoparticles mediate differential responses in keratinocytes and fibroblasts during skin wound healing. Chem Med Chem 2010;5:46875 .

18. Kim J.S., Kuk E., Yu K.N. Antimicrobial effects of silver nanoparticles. Nanomed Nanotechnol Biol Med 2007;3:95101.

19. Lansdown A.B. Silver. 1. Its antibacterial properties and mechanism of action. $J$ Wound Care 2002;11:125-130.

20. Beddy D., Watson R.W., Fitzpatrick J.M., O'Connell P.R. Increased vascular endothelial growth factor production in fibroblasts isolated from strictures in patients with Crohn's disease. Br J Surg 2004;91:72-7.

21. Rajan R, Chandran K, Harper SL, Yun SI, Kalaichelvan PT. Plant extract synthesized silver nanoparticles: an ongoing source of novel biocompatible materials. Ind Crops Prod 2015;70:356-73.

22. Mittal AK, Bhaumik J, Kumar S, Banerjee UC. Biosynthesis of silver nanoparticles: elucidation of prospective mechanism and therapeutic potential. J Colloid Interface Sci 2014;415:39-47.

23. Ovais M, Khalil AT, Raza A, Green synthesis of silver nanoparticles via plant extracts: beginning a new era in cancer theranostics. Nanomedicine (Lond) 2016;12(23):3157-3177.

24. Ahmed KBR, Nagy AM, Brown RP, Zhang Q, Malghan SG, Goering PL. Silver nanoparticles: significance of physicochemical properties and assay interference on the interpretation of in vitro cytotoxicity studies. ToxicolIn Vitro 2017;38:179-92.

25. Tak YK, Pal S, Naoghare PK, Rangasamy S, Song JM. Shape-dependent skin penetration of silver nanoparticles: does it really matter? Sci Rep 2015;5:16908.

26. Sun B, Barnard AS. The impact of size and shape distributions on the electron charge transfer properties of silver nanoparticles. Nanoscale 2017;9(34):12698-708.

27. Ravindran A, Chandran P, Khan SS. Biofunctionalized silver nanoparticles: advances and prospects. Colloids Surf B Biointerfaces 2013;10:342-52.

28. Divakar DD, Jastaniyah NT, Altamimi HG, Enhanced antimicrobial activity of naturally derived bioactive molecule chitosan conjugated silver nanoparticle against dental implant pathogens. Int J Biol Macromol 2018;108:790-7.

29. LiangpengGe, Qingtao Li, Meng Wang, Jun Ouyang, Xiaojian Li, and Malcolm MQ Xing et al. Nanosilver particles in medical applications: synthesis, performance, and toxicity. Int J Nanomedicine 2014;9:2399-2407.

How to cite this article: Yadav C, Rajat V, Goyal A. Silver nanoparticles used in the antibacterial preparations. Int J Pharm Chem Anal 2019;6(3):55-9. 